\title{
Antihumanismo o autonomía del individuo ante las estructuras sociales: La relación individuo-sociedad en la teoría de Niklas Luhmann
}

\author{
ANTI-HUMANISM OR AUTONOMY OF THE INDIVIDUAL VIS-A-VIS SOCIAL STRUCTURES: THE INDIVIDUAL- \\ SOCIETY RELATIONSHIP IN NIKLAS LUHMANN'S THEORY
}

Dra. Cecilia Dockendorff (ㄷocken@soles.cl) Instituto de Estudios Avanzados, Universidad de Santiago (Santiago, Chile)

\begin{abstract}
The individual-society relationship remains a central issue in the social sciences which has not yet reached a consensual explanation. This article presents the way in which Niklas Luhmann's theory of social systems deals with the subject. I discuss some of the critical approaches this theory has arises. Then I present social system's concepts and partial theories that describe the individual-society relationship. I conclude with some reflections about what we consider to be "theoretical advantages" regarding Luhmann's theory vis-a-vis common explanations in sociology. This, because in Luhmann's theory of social systems -contrary to critical judgments-, individuals are theoretically placed in society's environment, thus maintaining a relative autonomy which allows them to consider without idealism their actual possibilities to influence society.
\end{abstract}

Key words: sociology, individual, systems theory, Luhmann, humanism

\section{Resumen}

La relación individuo-sociedad continúa siendo un problema central en las ciencias sociales, sin haber encontrado aún una solución teórica que alcance un mínimo consenso. Este artículo discute el abordaje al tema por parte de la teoría de sistemas sociales propuesta por Niklas Luhmann, considerando algunas de las críticas que ha suscitado. Se presentan los conceptos y teorías parciales con que la teoría de sistemas sociales describe la relación individuosociedad. Se concluye con una reflexión sobre lo que se pueden considerar "ventajas teóricas" respecto de la teoría luhmanniana en oposición a explicaciones tradicionales de la sociología, por cuanto, contrario a los juicios críticos, en la teoría de sistemas sociales los individuos concebidos teóricamente en el entorno de la sociedad conservan una autonomía relativa que les permite evaluar, sin idealizaciones, sus posibilidades concretas de influir en ella.

Palabras clave: sociología, individuo, teoría de sistemas, Luhmann, humanismo

\section{Introducción}

La reflexión sobre la relación individuo-sociedad en la historia del pensamiento occidental se remonta hasta Aristóteles y su distinción entre las relaciones de los individuos entre sí (ética) y las relaciones entre el individuo y la sociedad (política) (1). En el pensamiento sociológico ha sido tema insoslayable, abordándose en base a diversas 
orientaciones teóricas, las que a pesar de haber dado pasos decisivos, aun no se muestran concluyentes. Desde Durkheim no se ha cuestionado la cualidad sui géneris de la sociedad; realidad social que ya Marx considerara la fuente histórica de alienación de los individuos, cuya libertad y capacidad de elegir se limita por determinaciones materiales y sociales, ante lo cual están llamados a luchar colectivamente. Weber por su parte, en base a su "método individualista" inaugura una deriva teórica que continúa hasta nuestros días, dispuesta a revindicar la dimensión subjetiva del individuo ante las estructuras sociales. No han faltado los intentos por diluir la distinción entre individuo por una parte, y sociedad por otra, como es el caso de Elías, cuya propuesta afirma que "individuo" y "sociedad" no son dos objetos que existan de forma separada, sino solo dos planos analíticamente distintos pero inseparables de una misma realidad. Desde la sociología crítica, Adorno y Horkheimer cuestionan la tradición sociológica por utilizar un concepto de individuo que designa una singularidad nacida bajo el liberalismo y la doctrina de la libre competencia, destacando que las relaciones entre individuo y sociedad representan el tema central de la sociología, por lo que la fecundidad de las teorías sociológicas se mediría por la capacidad para penetrar en tales relaciones. Indicativa también de la importancia central que adquiere el tema para la sociología, es la propuesta de Wright Mills en cuanto a que la relación entre la sociedad y las mentalidades psíquicas merecería una disciplina académica especial, a la que bautizó como "sociotics", mientras llamó “imaginación sociológica" a la habilidad para captar la unidad entre la historia, la biografía y las estructuras sociales. En lenguaje contemporáneo y como parte de una controversia con Luhmann, Habermas ha expresado: "La relación entre individuo y sociedad, tan discutida en la vieja sociología se refiere a un problema de demarcación que hoy vuelve a plantearse en la cuestión de cómo se relacionan entre sí el sistema psíquico y el sistema social" (Habermas 2002:362).

Se observa así que no se trata de una relación fácil de abordar teóricamente, lo que da cuenta de que la sociología no ha podido encontrar una respuesta satisfactoria ni consensuada. El tema conlleva fundamentos epistemológicos y de metateoría sociológica que demandan tratamiento explícito. Aunque no se analizan esas dimensiones en este texto, se puede decir en todo caso que se intentará responder la pregunta que plantea Archer: los seres humanos conceptualizados en las diversas teorías sociales, ¿terminan siendo títeres o titiriteros en el escenario de lo social? Desde un paradigma de realismo analítico, Archer aborda la discusión de dichos problemas con los conceptos de "conflacionismo" y "elisionismo", cuestionando la disolución de las propiedades del individuo en la sociedad o viceversa. Sus conclusiones no dejan muy bien paradas a las teorías sociológicas tradicionales, desde los clásicos hasta Giddens. Archer fundamenta su propio aporte para superar dichos desequilibrios explicativos, en el concepto de emergencia. Por su parte, Mascareño (2008) parece corroborar los hallazgos de Archer al desarrollar un análisis comparativo entre Parsons, Coleman, Archer y Luhmann, en base al concepto de emergencia, destacando la solución sistémica en la perspectiva luhmanniana a los problemas de conflacionismos indicados por Archer.

Es en torno a este persistente tema teórico de la relación individuo-sociedad, que se plantean aquí los argumentos que llevan a considerar la solución que ofrece la teoría de sistemas sociales propuesta por Niklas Luhmann (1991, 2007). La particular descripción de los individuos y la sociedad que propone el autor, forma parte de las opciones básicas de la teoría de sistemas sociales, cuyas premisas teóricas basadas en un paradigma sistémico, que se apartan considerablemente de la tradición sociológica, cobran un alto precio de incomprensión y rechazo. Es lo que ocurre respecto de observar al individuo ubicado en el entorno de la sociedad. En efecto, la arquitectura teórica sistémica observa al individuo como un sistema psíquico, operativamente clausurado a la vez que cognitivamente abierto, que se ubica en el entorno de la sociedad. Como consecuencia, los individuos dejan de ser los actores o sujetos considerados las unidades básicas constitutivas de la sociedad, en tanto permanecen situados en el entorno de ésta.

La sociología no sistémica ha formulado críticas en cuanto a que ubicar a los individuos en el entorno de la sociedad implica otorgarles un papel secundario ante una necesaria primacía de las estructuras sociales. Dichas críticas van desde que propicia un modelo de sociedad hipostasiado en la idea de sistema (Habermas), hasta un antihumanismo metodológico que sustituye la acción colectiva por la comunicación, y sustrae con ello a los actores empíricamente observables de los procesos de decisión (von Reese-Schaefer).

Pero es posible sostener que, contrario a dichos juicios críticos, el concebir al individuo situado en el entorno de la sociedad ofrece "ventajas teóricas", esto es, la posibilidad de utilizar conceptos de mayor alcance explicativo que 
permitan comprender tanto su independencia como su relación con la sociedad. En efecto, el individuo en el entorno social conserva un rol protagónico en tanto origen o condición necesaria para la formación de los sistemas sociales, lo que le permite a la teoría de sistemas sociales proveer una noción clara de la autonomía de los individuos y posibilita así una mejor observación de su complejidad y, desde allí, su vínculo con la sociedad.

Asumiendo que tanto Luhmann como la mayoría de los sociólogos sistémicos se ha concentrado en la descripción del sistema social, dando la (falsa) impresión de que la teoría no entrega posibilidades teóricas suficientes para describir a los individuos, amén de dejarlos a merced de estructuras sociales auto-referentes, este artículo se propone discutir la teoría de sistemas sociales optando por la observación de los individuos y su relación con la sociedad.

El artículo está dividido en tres partes. En una primera se detiene en algunas de las críticas sociológicas respecto de la posición de los individuos en el entorno de la sociedad. Luego se exponen las distinciones sistémicas con que la teoría describe a los individuos y explica la relación individuo-sociedad. En una tercera parte, considerando las críticas esbozadas, se expone lo que se consideran "ventajas teóricas" por parte de la perspectiva sistémica, para describir a los individuos y resolver el persistente problema sociológico de su relación con la sociedad.

\section{El "escándalo" de los individuos fuera de la sociedad}

En La Sociedad sin Hombres, Niklas Luhmann o la teoría como escándalo, el filósofo español Ignacio Izuzquiza (1990) aborda la "radical perplejidad" en que la obra de Luhmann deja a los lectores cuyo pensamiento se enmarca en la tradición de la sociología y las ciencias sociales. Tal vez la perplejidad o el escándalo mayor sea el que en la obra de Luhmann los individuos pierden su calidad de elementos unitarios constitutivos de la sociedad, pasando a formar parte de su entorno. Escandaloso porque, como lo plantea Izuzquiza, es "algo que puede llevar a entender que la sociedad -o los sistemas sociales como son el derecho, la economía, la política, etc.- se levanta con sus propias reglas y nunca muestra una ternura añorada por las situaciones humanas" (1990:12).

Si ello ocurre así o no, resulta una apreciación que puede variar bastante según la perspectiva de cada observador. Lo que sí constituye una conclusión generalizada, y declarada por el propio Luhmann, es que la teoría de sistemas sociales se abstiene del estudio de la sociedad bajo la perspectiva humanista heredada del pensamiento ilustrado y que ha orientado la reflexión sociológica tradicional. Dice Izuzquiza: "Leer a Luhmann supone, en cierta medida, hacer una cura contra toda tentación de privilegio antropológico" (1990:12), y ello también implica una prescindencia de toda necesaria orientación normativa por parte de la teoría sociológica, lo que para muchos constituye una fuente adicional de escándalo.

Pero, ¿qué tan escandaloso resulta para la comprensión de la relación individuo-sociedad, en particular para esclarecer la propia ubicación del individuo ante las estructuras sociales, el que teóricamente se lo ubique en el entorno de la sociedad? Dicho de otra manera, ¿qué tan antihumanista resulta el no humanismo de Luhmann?

Se sabe que tanto Habermas como Luhmann admiten la crisis de la llustración y su confianza en un racionalismo que requiere de ser superado. Mientras Habermas sostiene que dicha superación consiste en recuperar el lugar del mundo de la vida que la racionalidad científica y técnica habría excluido, Luhmann, de cara a la creciente complejidad que alcanza la sociedad bajo el primado de la diferenciación funcional, se abstiene de integrar la crítica en su teoría de lo social. Es precisamente esta ausencia de crítica integrada a la descripción de la sociedad lo que conduce a que Habermas termine observando una indiferencia hacia lo humano por parte de Luhmann, y es este supuesto antihumanismo luhmanniano uno de los puntos centrales de la confrontación que sostuvieran Luhmann y Habermas.

Pero si, como propone Izuzquiza, se deja de lado el prejuicio de tildar a Luhmann de conservador porque no se presenta como un continuador de la tradición emancipatoria heredada de la concepción humanista ilustrada, se puede descubrir si la teoría de sistemas sociales deja en una situación desmedrada al individuo frente a la sociedad, privado de su capacidad de incidencia, o si, por el contrario, aclara mejor las condiciones que dicha incidencia debe enfrentar en la compleja sociedad contemporánea. 
Lo que Luhmann describe acríticamente es condenado por Habermas como un objetivismo funcionalista que deja fuera la intersubjetividad dialógica del mundo de la vida, en una sociedad concebida como "sin cabeza". Alude de esta manera al carácter acéntrico de la sociedad funcionalmente diferenciada, que para Luhmann tiene como consecuencia que ya no pueda fijarse ningún punto de vista desde el cual observar correctamente el todo, ya se le llame Estado o sociedad. Habermas no acepta dar por perdida la posibilidad de una autorrepresentación de la sociedad, la que concibe en los espacios públicos entendidos como intersubjetividades de orden superior. Sostiene Habermas: "En ellos pueden articularse auto-calificaciones y auto-atribuciones colectivas formadoras de identidad. Y en el espacio público de orden aún más superior, resultante de la agregación de los anteriores, puede también articularse una conciencia social global" (1993:443).

Por cierto, en la arquitectura teórica de Luhmann, la unidad de la sociedad moderna se presenta de forma variada desde las perspectivas de sus distintos subsistemas; no puede darse una autoconciencia que abarque a la sociedad entera. Habermas insiste en que si desde la perspectiva sistémica las sociedades modernas no tienen la posibilidad de desarrollar una identidad racional, entonces falta todo punto de referencia para una crítica a la modernidad, y, en el caso de que la hubiera, la crítica estaría condenada al fracaso ante la diferenciación que discurre a espaldas de la razón.

Pero se puede argumentar que aunque resulta claro que la diferenciación efectivamente ocurre a espaldas de alguna supra-razón, ello constituye justamente la condición de posibilidad de adoptar no sólo un único punto de referencia para criticar la modernidad sino múltiples y diversos. Las críticas venidas desde el entorno de la sociedad pueden multiplicarse y diversificarse, sin que el "espacio público" se ordene jerárquicamente en un "orden superior" para llegar a "una conciencia social global".

Adicionalmente, Habermas refuerza su argumentación en contra del lugar secundario en que la teoría de sistemas sociales ubicaría a los individuos, criticando la opción luhmanniana por adoptar el concepto de observador en remplazo del concepto de sujeto. Le critica a Luhmann que presuponga sencillamente que las estructuras de la intersubjetividad se desmoronan, que los individuos hayan sido arrancados de su mundo de la vida y que el sistema de la personalidad y el sistema social constituyan apenas entornos el uno para el otro, mientras los propios sujetos degeneran en sistemas.

Al respecto se puede argumentar que efectivamente el individuo, en tanto sistema psíquico, es definido por la teoría luhmanniana como un sistema observador que efectúa distinciones. Ello le permite a Luhmann abandonar el tradicional concepto de sujeto el que, según él, le resta potencial explicativo a la tradición sociológica en general. En su argumentación, el concepto de observador permite escapar a cualquier fundamento universal del conocimiento en tanto la distinción es siempre una operación empírica, que puede conducir a un conocimiento tanto verdadero como falso. De modo que, efectivamente, el concepto de observador no cuenta con una racionalidad universal garantizada que lo oriente en sus comunicaciones.

Esta consecuencia que trae el adoptar el concepto de observador despojado del humanismo positivo, es una de las que suscita fuertes observaciones críticas por parte de Habermas. Desde su perspectiva, el recurrir al concepto de observador implica despojar de todo asiento racional al individuo, amén de que éste perdería sus derechos al volverse objeto de la contingencia que caracteriza a una sociedad sin cabeza.

Desde luego, es posible reconocer que, en efecto, en la teoría luhmanniana todo queda librado a la variación y la contingencia, pero se puede argumentar que una sociedad sin cabeza y sujeta a la contingencia no necesariamente les quita derechos a las operaciones intencionales de los individuos, en la expresión de Habermas. Muy por el contrario, las operaciones intencionales de los individuos (las variaciones introducidas en la comunicación por los sistemas psíquicos, en lenguaje sistémicamente), forman parte de aquella contingencia y lo que los individuos pierden en términos de la importancia central que les otorga el humanismo, lo recuperan desde capacidad de incidencia factual en la sociedad que adquiere su operar como sistemas psíquicos acoplados a los sistemas sociales. 
Al respecto cabe destacar que la decisión teórica de conceptualizar a los individuos como sistemas observadores situados en el entorno de la sociedad, no hace necesarios puntos de referencia normativos a priori, como en la teoría de la acción comunicativa de Habermas, sino que se trata de observar las posibilidades concretas de que las irritaciones realizadas por los individuos logren abrirse camino en la contingencia comunicativa de la sociedad. En efecto, tales posibilidades no adquieren su fuerza de alguna racionalidad supra-individual, sino de la capacidad de estructuración comunicativa que los individuos, asociados o no, logren adquirir en el propio proceso de comunicarse, no necesariamente hacia el entendimiento. Se puede acceder así a una multiplicidad de racionalidades que buscan (o luchan por, al decir de Bajtin) ser reestabilizadas en la comunicación social.

En la línea argumental crítica de Habermas, pero intentando dilucidar el antihumanismo de Luhmann, Patricio Miranda (2012) busca descubrir las raíces antropológicas de dicho antihumanismo teórico del autor. Las encuentra en la tradición de la antropología filosófica alemana, desde Max Scheler a Helmut Schelsky. Las conclusiones de su estudio apuntan a develar una concepción de lo humano subyacente, y tal vez poco asumida por el propio Luhmann en su afán por descartar el humanismo positivo que había inspirado a la sociología hasta entonces. Miranda establece con claridad que no hay teoría sociológica posible sin una concepción subyacente de lo humano. La teoría luhmanniana no escaparía a ello, por lo que ante su negativa de adoptar un humanismo positivo, lo que le queda como opción aparece como un humanismo negativo, precisamente el sostenido por la antropología filosófica alemana. ¿En qué consiste? Básicamente en concebir al ser humano como un ser constitutivamente desarraigado en el mundo; mundo social que se resiste a ordenarse según sus propósitos y valores. Deviene así un solucionador de problemas, un ser humano enfrentado a la contingencia que su propio afán comunicativo va haciendo emerger en la sociedad, y que no tiene garantizada la consecución de sus más caros propósitos. Ni la filosofía ni la historia le ofrecen esa seguridad. Esta concepción negativa de ser humano lo retrata como un ser problemático ante sí mismo, un ser abierto al mundo (Scheler), un ser carencial (Gehlen), descentrado y objetivado respecto de su cuerpo (Plessner).

Este humanismo negativo no es, por cierto, privativo de la teoría de sistemas sociales de Luhmann. Se encuentra en todos los críticos de la razón, desde Nietzsche y sus sucesores (entre los cuales Habermas sitúa a Luhmann). El humanismo negativo es asimismo propio de las corrientes existencialistas, en las que también Habermas incluye a Luhmann, atribuyéndole dos supuestos antropológicos básicos: “Por un lado, Luhmann comparte las suposiciones básicas de una antropología existencialista de tipo sartriano. La realidad es concebida, conforme al principio 'todo es posible' (...) Por otro lado, Luhmann comparte la convicción fundamental de la antropología institucionalista de procedencia gehleniana" (Habermas 2002:322).

Cabe destacar que, a diferencia del humanismo negativo sartriano, nietzscheano, el de Sloterdijk o el de la antropología filosófica alemana, en los que es dable encontrar algún juicio normativo respecto de la situación humana, en el caso de Luhmann solo se trata de la negativa a incorporar en la descripción de lo social el humanismo positivo que caracteriza a la tradición sociológica. Sólo en esto consiste el antihumanismo luhmanniano, en rechazar que la sociedad sea la variable dependiente resultante del efecto de la variable independiente de la acción (comunicativa o no) y/o interacción humana (Miranda 2012:279). Es el concepto humanístico de sociedad lo que Luhmann abandona, no así una -metateóricamente inevitable-concepción del ser humano, el que al interior de la teoría de sistemas sociales se puede observar en su propia autonomía y su capacidad relativa y realista de influir en el acontecer de la sociedad. Pero Luhmann no se pronuncia normativamente ante esta situación; no la propone como favorable para un proyecto humano común; no construye la teoría incorporando ese horizonte normativo. El humanismo negativo de Luhmann vale para la descripción sociológica de la sociedad, descripción que la sociología crítica realiza aferrada, contra toda evidencia insiste Luhmann, a un concepto de sociedad que tiene su referencia esencial en el individuo. Ello es lo que alimenta la ilusión de que la sociedad pueda organizarse de modo humano, esto es, sea entendida como un epifenómeno de la actividad intencionalidad de los individuos. Esta ilusión es la que Luhmann rechaza como fundamento de su teoría, y que la hace prescindir de cualquier indicación normativa de la situación humana y su destino.

Desde la perspectiva de la relación individuo-sociedad, la teoría de sistemas sociales que obliga a desprenderse de esa ilusión de que la sociedad pueda organizarse de modo humano, no ofrece más que la condición de posibilidad 
para observar, en forma no idealizada, la situación del individuo ante la sociedad. El antihumanismo de Luhmann no hace sino resaltar la necesidad de asumir dicha situación, y desde ella evaluar las posibilidades concretas que tienen los seres humanos de influir en la sociedad, precisamente porque ésta no se orienta a partir de un humanismo positivo garantizado.

Se puede sostener así que, contrario a lo que podría esperarse, la ubicación de los individuos en el entorno de la sociedad facilita, sin sesgos idealizadores, la observación de la relación individuo-sociedad, sus propiedades diferenciales en la constante construcción de lo social. Es precisamente esta premisa sistémica, entre otras, la que otorga sustento a la idea de "ventaja teórica" a la hora de comprender los alcances de la relación individuo-sociedad en ambos sentidos de condicionamiento mutuo.

En síntesis, la ubicación de los individuos en el entorno de la sociedad no constituye per se un problema teórico. Donde se podría encontrar, sin embargo, algo que podría llamarse una "negligencia teórica" (falta de tratamiento teórico equilibrado) por parte de la perspectiva luhmanniana, no es en relación a un desconocimiento de la contribución de los individuos a la morfogénesis de lo social, sino que aparece en el plano de la exposición de la teoría, en tanto muestra un poco equilibrado tratamiento del operar autónomo de los individuos en comparación con la extensa descripción del operar de la sociedad.

Dicha "negligencia teórica" puede observarse en que, a pesar de que la teoría de sistemas sociales plantea que sistema y entorno se implican mutuamente, por lo que la emergencia del sistema de la sociedad no puede explicarse sin referencia a los individuos como uno de sus entornos, el tratamiento de los propios individuos no ha estado aparejado al tratamiento dado al nivel de la sociedad. Es más, a pesar de que Luhmann ha planteado que no se pueden observar separadamente sistema y entorno, por lo que ambos -individuo y sociedad- están presentes en el nivel emergente de la comunicación, el propio concepto de comunicación es usado en la teoría para hacer referencia al sistema social.

Por cierto, únicamente los sistemas sociales incluyen y excluyen las comunicaciones que les corresponden, pero la teoría pone el acento en la selección por parte del sistema social y no en las posibilidades irritatorias de los sistemas síquicos que se incluyen en las comunicaciones de los diferentes sistemas sociales. Está claro que el sistema incorporará bajo sus propios términos comunicaciones que le hagan sentido a su lógica sistémica, pero destacar mayormente la lógica sistémica por sobre la participación de los sistemas síquicos en la comunicación termina facilitando un sesgo interpretativo que contribuye a la crítica generalizada de otorgar un papel secundario a los individuos y subordinarlos al funcionamiento de la sociedad.

Se puede ver así que el problema de la falta de centralidad del individuo en la teoría de sistemas sociales no es imputable a la arquitectura misma de la teoría, sino que a una contingente opción por describirla desde la perspectiva de la sociedad y no desde la referencia sistémica de los individuos. En efecto, si bien el sostener que los individuos están en el entorno de la sociedad no reduce su complejidad, precisamente porque al estar en el entorno de lo social los individuos no dependen de la sociedad, la autonomía de éstos y su complejidad no han sido suficientemente tematizadas por la teoría de sistemas sociales. Ello implica un desafío teórico pendiente, abierto a quienes, ya sea que como consecuencia de una deficiente exposición por parte de la sociología sistémica, o en virtud de hacerse eco de una crítica prejuiciosa, no han aquilatado lo que la teoría de sistemas sociales tiene para ofrecer como comprensión del individuo y su relación con la sociedad.

\section{Individuo, persona y semántica de la individualidad}

Se presentan a continuación los conceptos sistémicos con que la teoría describe a los individuos y aquellos que permiten dar cuenta específica del vínculo del individuo con la sociedad.

La opción por describir al individuo desde la teoría de sistemas sociales de Luhmann implica un primer ejercicio de apertura cognitiva para comprender al individuo como un sistema psíquico -acoplado a un sistema orgánico-y a la sociedad como un orden emergente de sistemas de comunicación. Ambos tipos de sistemas son indicados por la 
teoría como operativamente clausurados pero acoplados a través de diversos mecanismos. La distinción de niveles de emergencia sistémicos establece que los sistemas psíquicos, asiento de la individualidad, y los sistemas sociales están separados pero íntimamente relacionados en tanto constituyen entornos los unos de los otros y no pueden operar sino mutuamente acoplados.

La alta abstracción que caracteriza a esta teoría y su poca tradicional forma de describir la individualidad no le impide, sin embargo, reconocer una básica y común referencia empírica. Luhmann expresa este reconocimiento del individuo al afirmar: "Se puede partir de la base de que la individualidad del hombre -comprendida como una unidad corporalpsíquica en el sentido de su automovilidad y, sobre todo, en el sentido de la muerte propia y única de cada uno- es una experiencia aceptada y reconocida por todas las sociedades" (1985:15). Sin duda la concepción de la individualidad en la teoría de sistemas sociales, por poco convencional que se muestre, no trasgrede esta evidencia empírica.

Hecha esta salvedad, se puede abordar la individualidad desde los conceptos utilizados por la perspectiva sistémica. Para ello se debe partir por una primera distinción a desarrollar a continuación: individualidad como concepto teórico e individualidad como una semántica histórica.

Como concepto teórico, la teoría de sistemas sociales aborda la individualidad del ser humano utilizando los conceptos de sistema psíquico y persona. Estos son los conceptos teóricos que permiten indicar la individualidad, más claramente al ser humano, conceptos que se diferencian tanto de conceptos sociológicos tradicionales -sujeto, actor, agencia-como también de los atributos y características con que se ha especificado históricamente a los seres humanos. Dichos atributos constituyen para la teoría de sistemas auto-descripciones (2) que realiza la sociedad para comprender a los seres humanos, a las que la teoría observa como semánticas históricas, y no utiliza en calidad de conceptos teóricos.

\section{Sistema psíquico}

Al interior de la teoría sistémica, los individuos se entienden como sistemas de conciencia (y de vida) cerrados, autónomos y autorreferentes, teóricamente definidos como sistemas autopoiéticos. Es la autopoiesis del sistema psíquico la que define la individualidad, en tanto clausura de su auto-reproducción circular. Respecto a la teoría de la conciencia que se desprende de la conceptualización de los sistemas psíquicos, la teoría de sistemas sociales logra a la vez hacer justicia a la referencia empírica mencionada anteriormente como asimismo superar los problemas filosóficos derivados de la concepción trascendental de la conciencia, problemas que, según Luhmann, permanecían sin solución concluyente en las ciencias sociales. Luhmann desarrolla su argumentación en torno al problema filosófico de la trascendentalidad de la conciencia (3), sosteniendo que sistémicamente por conciencia no debe entenderse algo que existe sustancialmente, sino que se trata sólo del modo de operación específico de los sistemas psíquicos (1991:268).

Conviene volver a destacar que la autopoiesis del sistema psíquico requiere de condiciones externas, las que vienen dadas por las estructuras sociales. Sistémicamente, el ser humano sólo puede ser individuo con base en la clausura operativa y en la auto-reproducción de sus propias vivencias, pero la sociedad, como su entorno, le provee estructuras para mantener activa su operación autopoiética. ¿Cómo lo hace? ¿Con qué distinciones teóricas da cuenta la teoría de sistemas sociales de la relación entre sistemas psíquicos y sociales? Los conceptos de expectativas, acoplamiento estructural, las teorías de la comunicación y de los medios de comunicación simbólicamente generalizados explican sistémicamente dicha relación. Se exponen sucintamente a continuación.

Desde su operar autopoiético, el sistema psíquico observa su entorno y establece una relación de acoplamiento con éste por medio de estructuras que la teoría describe como expectativas. El concepto de expectativa alude a condensaciones de referencias de sentido cuya función es orientar de modo relativamente estable la comunicación y el pensamiento frente a la complejidad y contingencia del mundo. Tal vez se aclare este concepto en la siguiente expresión gráfica que utiliza Luhmann: “En cuanto a los sistemas psíquicos, entendemos por expectativa una forma 
de orientación por medio de la cual el sistema sondea la contingencia de su entorno en relación consigo mismo y la acoge como incertidumbre propia en el proceso de la reproducción autopoiética" (1991:273).

Las expectativas, entonces, son las estructuras que predisponen a los sistemas a la relación con su entorno. Operan en ambos sentidos: del sistema psíquico a los sistemas sociales (interacciones, organizaciones o sistemas funcionales) y vice-versa. Lo que se produce como cruce de tales expectativas es el acoplamiento estructural en forma de comunicación.

La teoría de la comunicación de Luhmann parte sosteniendo que el éxito de la comunicación resulta extremadamente improbable; más aún si, además de estar operativamente clausurados, los sistemas autopoiéticos se encuentran en una situación de doble contingencia (se trata este concepto en el siguiente apartado). La teoría de la comunicación luhmanniana presenta particularidades que la diferencian de las teorías tradicionales de la comunicación, en especial de aquellas basadas en la transmisión de información de alter a ego. Luhmann describe la comunicación como un proceso de tres selecciones, las que deben enfrentar la contingencia tanto universal como subjetiva. Así, desde su clausura operativa, alter efectúa dos selecciones: la información que desea comunicar a ego, y una forma particular de hacerlo (en alemán Mitteilung, que ha sido traducido como conducta o acto de notificación, de participación, de dar a conocer, y que correspondería al concepto tradicional de acción). Por su parte ego, debe primero atribuir a alter su conducta (como una vivencia o una acción) (4) y a partir de ella distinguir la información (contingencia universal) de la conducta de notificación de ego (contingencia subjetiva) y, en base a su propia clausura operativa, a través de su propia vivencia o acción, comprender. Ahora, la clausura operativa y el diferencial constitutivo y biográfico de cada individuo participante en la comunicación, implican que la comprensión de ego de la oferta comunicativa de alter sea apenas probable.

Ante ello, la teoría plantea que la sociedad ha establecido, evolutivamente, mecanismos que aumentan la probabilidad de comprensión y éxito de la comunicación. Junto al lenguaje, el medio primario de acoplamiento conciencia-comunicación, se estandarizan estructuras de expectativas y medios simbólicos que anteceden y facilitan la conectividad entre alter y ego. En efecto, se requiere más que el lenguaje para garantizar el acoplamiento estructural entre sistemas psíquicos y sociales. Así, evolutivamente se han desarrollado medios de difusión que amplían el círculo de receptores de la comunicación, como la escritura y la imprenta. Estos superan la dependencia de la comunicación al espacio y al tiempo; últimamente los medios electrónicos logran llevar casi a cero las limitaciones espaciales y temporales de la comunicación, junto a suministrar posibilidades técnicas de registro y almacenamiento de comunicaciones (Luhmann 2007:234). Pero los medios de difusión aún son insuficientes para motivar el acoplamiento conciencia-comunicación y lograr el éxito (continuidad ininterrumpida) de la comunicación. La teoría destaca como el mecanismo moderno por excelencia que logra acoplar la motivación individual con la selección social, a los medios de comunicación simbólicamente generalizados (2007:156).

La teoría de los medios de comunicación simbólicamente generalizados constituye uno de los pilares teóricos de la teoría de sistemas sociales para dar cuenta del vínculo entre el individuo y la sociedad, en la compleja sociedad moderna. Esta teoría explica cómo a través de esta estructura de coordinación de la motivación individual y la selectividad social -los medios simbólicos- se plausibiliza el acoplamiento conciencia-comunicación en la sociedad funcionalmente diferenciada. Dichos medios simbólicos, en la actualidad la propiedad (el dinero), el poder (y la legalidad), la verdad, el arte y el amor, logran alentar la comunicación proveyendo oportunidades para que sea aceptada: tratan de hacer posible comunicaciones que son improbables. Su función consiste en volver objeto de expectativa la aceptación de una comunicación en los casos en que es probable el rechazo. Se profundiza algo más en la teoría de los medios de comunicación simbólicamente generalizados en el próximo apartado.

Si bien la teoría de sistemas sociales describe la individualidad en su unidad como un sistema psíquico, desde la perspectiva de la sociedad, entendida ésta como el nivel emergente de la comunicación que se diferencia según niveles de constitución de sistemas interaccionales, organizacionales y funcionales, el sistema psíquico pasa a ser entorno de la sociedad y es observado por ésta en calidad de persona. 


\section{El concepto de persona}

A diferencia de los sistemas psíquicos, que indican sistemas autopoiéticos que operan distinguiendo constantemente auto-referencia de hétero-referencia, las personas no constituyen sistemas. No indican al ser humano como un todo, tanto corporal como espiritualmente (Luhmann 1998b:236). Ocurre que en el acoplamiento concienciacomunicación, los sistemas sociales requieren identificar a quienes participan en el suceso comunicativo. Es a la forma de esta identificación a la que Luhmann llama persona (2007:77) (5). ¿Por qué se produce la forma persona y cuál es su función? El autor responde que para que las expectativas mutuas y las conductas de los participantes aumenten sus posibilidades de inteligibilidad, es necesario que se limite el repertorio de posibilidades. Evolutivamente ello se muestra así, desde las formaciones sociales más simples en las que el atavío de los cuerpos expresaba limitaciones sociales específicas, hasta la compleja sociedad moderna en la cual la diferenciación de sistemas funcionales tipifica los contextos sociales a los que los sistemas psíquicos se acoplan en calidad de personas, por ejemplo como pacientes, clientes, alumnos, consumidores, artistas, ciudadanos.

Los sistemas sociales -sean interacciones, organizaciones o sistemas funcionales- "personalizan" sus referencias externas, las cuales, a través de la reutilización de estas referencias, "condensan" a las personas, las dejan fijas como si fueran idénticas, las confirman, las enriquecen con nuevas referencias (Luhmann 2007:78). A través de la comunicación que le es propia, cada sistema puede incluir o excluir a los sistemas psíquicos, en tanto se refiere a éstos en calidad de personas. Son estas referencias externas las que cumplen la función de limitar posibilidades, designadas con el concepto de inclusión, o más bien con la distinción inclusión/exclusión (6).

Desde la perspectiva de los individuos, cabe destacar que en las sociedades estamentales, la inclusión de los seres humanos estaba garantizada desde su nacimiento, y su ubicación sólo podía concebirse en un estrato específico. La inclusión se concretaba mediante una única posición social, por lo que los seres humanos podían estar afectados de muchos problemas pero no así del problema específico de quién se era, sostiene Luhmann (1995:63). En dichas sociedades este problema no podía surgir simplemente porque se era alguien por el hecho de ser miembro de la sociedad. La inclusión de los seres humanos cambia sustancialmente en la sociedad moderna bajo el primado de la diferenciación funcional: se convierte, según Luhmann, en un problema (1995:63). Efectivamente, la inclusión en la sociedad funcionalmente diferenciada se hace problemática en tanto el individuo deja de pertenecer a un solo sistema parcial de la sociedad y ésta ya no puede ofrecerle una única forma para incluirlo, sino múltiples. Se podría decir que la sociedad funcionalmente diferenciada hace una especie de disección del ser humano en diversas personas, en tanto los sistemas sociales incluyen personas pero no seres humanos.

\section{Semántica de la individualidad}

Un último concepto sistémico que refiere a la individualidad es la semántica de la individualidad. Sistémicamente, dado que la sociedad es entendida como comunicación, las referencias que circulan respecto de los individuos en la sociedad son observadas como semánticas históricas que los describen. A diferencia del concepto teórico sistémico que indica que "la individualidad no puede ser otra cosa que la cerradura circular de su reproducción autoreferencial" (Luhmann 1991:269), la sociedad por su parte utiliza referencias al ser humano en la comunicación, las que constituyen para la teoría de sistemas sociales auto-descripciones, esto es, semánticas que refieren y conceptualizan, en una variabilidad constatable históricamente, a los seres humanos.

Es así como el advenimiento de la modernidad resignifica la noción de individualidad: de indivisibilidad a singularidad. Efectivamente, Luhmann destaca que la acumulación de semánticas que remiten a la individualidad a comienzos de la modernidad se estabiliza cuando, en las postrimerías del siglo XVIII, se introduce definitivamente el término “individuo" (1995:76). Y es particularmente la semántica histórica del sujeto, la que da respuesta a qué es el ser humano en la sociedad moderna. El individuo se concibe como sujeto y así puede producirse su auto-afirmación y hacerse accesible a sí mismo en la reflexión (Luhmann 2007:812).

De este modo entonces, es la semántica del sujeto (sistémicamente una semántica histórica, no un concepto teórico) la que sostiene la potencial inclusión de todos los individuos en cualquiera de los sistemas diferenciados. Es 
precisamente la figura del sujeto, la que tiene la función de dar fundamento a la inclusión de todos en la sociedad. El ser humano deja de ser "individuado" ("individuiert") por estratos, dice Luhmann, y la sociedad moderna, que confía la regulación de las inclusiones a la autonomía de los sistemas funcionales, ya no puede excluir a ninguna persona (2007:812). En la sociedad moderna el ser humano es "individuado" universalmente con el concepto de sujeto. En la expresión de Luhmann: "el sujeto se ofrece como fórmula salvadora para que el modus de inclusión se traslade a las condiciones específicas modernas de los sistemas funcionales" (2007:813).

Los párrafos anteriores permiten concluir que la teoría de sistemas sociales cuenta, por una parte, con conceptos teóricos que permiten una clara descripción sociológica de los individuos y su vínculo con la sociedad, y, por otra, ofrece una comprensión de la noción de "individuo" en tanto semántica histórica, como una descripción históricoempírico (una auto-descripción), que la sitúa en la contingencia de la evolución social y que libera a la teoría de toda proposición normativa sobre lo que es o cómo debe ser el individuo (aunque, como lo recuerda Miranda, no pueda prescindir de una antropología subyacente). Ello permite concluir, que lo que se indica como "individualidad" en su versión moderna, es una semántica resultado de la evolución de la comunicación social, y su contenido no es ni más relevante ni más verdadero que la semántica con que se describía al ser humano en épocas anteriores, o de como se lo describirá eventualmente en el futuro.

\section{Las "ventajas teóricas" de observar a los individuos en el entorno de la sociedad}

La conclusión anterior nos permite señalar que la teoría sistémica y en particular el concepto teórico de sistema psíquico como descripción del asiento de la individualidad-que se relaciona con su entorno a través de acoplamientos estructurales-, hace innecesario recurrir a la descripción tradicional del vínculo entre el individuo y la sociedad en base al concepto teórico de sujeto y su concepto asociado de intersubjetividad, como tampoco requiere de los conceptos tradicionales de socialización e internalización. ¿Qué se gana con ello?

Se puede argumentar que las alternativas sistémicas a dichos conceptos tradicionales ofrecen explicaciones más plausibles, en tanto evitan los problemas asociados a éstos y que otras teorías sociológicas no han logrado superar. A partir de ello es que es posible hablar de "ventajas teóricas", como se aclarará a lo largo de este apartado.

Así, se puede indicar una primera ventaja teórica de utilizar la teoría de sistemas sociales para explicar la relación individuo-sociedad, en que permite resolver los problemas meta-teóricos de conflacionismo, ya sea ascendente (el individuo extiende sus propiedades a la sociedad) o descendente (la sociedad impone sus estructuras a los individuos), que plantea la distinción agencia/estructura (Archer 2009). En efecto, al separar teóricamente individuo y sociedad por medio del concepto de emergencia, la perspectiva sistémica logra mantenerlos teóricamente separados dejando en igualdad de condiciones de autonomía relativa tanto al individuo y su capacidad de incidencia sobre la sociedad, como la de ésta sobre los individuos. Ello permite reconocer que ambos, desde sus respectivas autonomías, aportan por igual al proceso de construcción de lo social, sin que pierdan ni impongan sus respectivas propiedades.

Si se observan dichas respectivas autonomías desde la perspectiva del individuo, la teoría de sistemas sociales parte reconociendo que son los individuos, en tanto sistemas psíquicos autopoiéticos que se observan mutuamente, los que ponen en marcha el sistema comunicacional y lo hacen posible. Esto implica otorgarles un rol protagónico en la emergencia de la comunicación. Los sistemas síquicos no pueden convertirse en parte del sistema social, pero son un requisito para la emergencia de los sistemas sociales. Esto significa que sin inclusión de los sistemas psíquicos no emerge ningún sistema social, pero ello implica reconocer también que es la evolución del sistema de la sociedad lo que regula la forma en que esta inclusión se hace posible. De este modo, individuo y sociedad mantienen su recíproca relación como sistemas y entornos mutuos (si se observan como lo uno o lo otro depende de la referencia sistémica que se elija) sin que se subordinen uno al otro, ni se diluyan (elisionismo) el uno en el otro.

Ahora, si se parte de la pregunta por cómo es posible un orden social que descansa en los individuos, la conceptualización de niveles de emergencia de sistemas autopoiéticos relacionados a través de una dinámica de acoplamientos estructurales, presenta una segunda ventaja teórica. Esta vez respecto de dar cuenta de los aspectos 
operativos necesarios para describir con claridad la relación individuo-sociedad. Frente a las descripciones de los procesos operativos propuestas por la teoría de sistemas sociales, conceptos tradicionales como los de cultura, socialización, intersubjetividad e integración social, se muestran carentes de capacidad explicativa en cuanto a la operatividad que suponen. En efecto, el concepto de cultura ha sido cuestionado por no lograr dar cuenta de fenómenos complejos y cada vez más diversificados en la sociedad contemporánea, los que ya no logran explicarse en base a invocar la cultura y su determinación sobre los individuos. Lo propio ocurre con los conceptos de socialización e intersubjetividad, conceptos que no especifican los aspectos operativos que llevan asociados. ¿Qué procesos de transmisión presuponen? Por su parte, el tema de la integración social no ha logrado superar los problemas de conflacionismo descendente asociados a los conceptos de norma y cultura.

Se puede observar esta superación de los conceptos tradicionales que logra la arquitectura sistémica en la reformulación que realiza Luhmann respecto de los conceptos parsonianos de doble contingencia, socialización y la teoría de los medios simbólicos. En ello se puede apreciar cómo se logran superar tanto los problemas teóricos de conflacionismo (en el caso de Parsons de tipo descendente) como los de insuficiente explicación de los mecanismos operativos involucrados.

Se ha mencionado respecto de la relación individuo-sociedad que en la perspectiva sistémica luhmanniana los medios simbólicos aparecen como el mecanismo moderno por excelencia que permite integrar conciencia y comunicación. Construyendo sobre las formulaciones de Parsons pero a la vez diferenciándose, Luhmann desarrolla su teoría de los medios de comunicación simbólicamente generalizados basada en la teoría de la doble contingencia, utilizando la distinción acción/vivencia de alter y ego y sus posibilidades de combinación. Es posible identificar aquí un avance teórico que surge del punto de partida que adopta Luhmann para reformular la teoría de los medios simbólicos, los que pasan de medios de intercambio entre sistemas sociales en la concepción parsoniana, a medios de comunicación simbólicamente generalizados. Luhmann formula su teoría de los medios de comunicación simbólicamente generalizados a partir del intento por comprender, precisamente, el vínculo entre la experiencia cotidiana de las vivencias y acciones de los individuos y los efectos evolutivos de la formación de estructuras sociales. El autor busca "unir más directamente el concepto de los medios generalizados con el problema central de la contingencia subjetiva de la orientación y de la elección" (1998a:12). Es esta formulación del acercamiento luhmanniano -un decisivo giro a partir de la teoría parsoniana- lo que le otorga una ventaja teórica a su teoría de los medios simbólicos, en tanto permite vincular dichos medios con la experiencia subjetiva. Ello implica que la propuesta luhmanniana del acoplamiento individuo-sociedad permite una comprensión de este vínculo logrando evitar la necesaria subordinación del individuo a la sociedad que se aprecia en la obra de Parsons.

Efectivamente, la construcción parsoniana muestra una desequilibrada participación por parte de los individuos y de la sociedad en el vínculo que se establece entre ambos. Al respecto, Mascareño (2009) señala cómo en Parsons los medios simbólicos de intercambio desarrollan una interpenetración asimétrica con la personalidad, por lo que aparece restringida la autonomía de la acción del individuo. Ello porque a nivel del sistema general de la acción, la interpenetración del sistema de la personalidad con el sistema social y la cultura revelan una subordinación cibernética de la personalidad tanto al sistema social como a la cultura, lo que obliga a Parsons a observar como conducta desviada la acción que no se somete a normas institucionalizadas. Esta asimetría es lo que le resta potencia a la acción individual como fuente importante de variación institucional o normativa.

Por el contrario, dicha asimetría se resuelve, o más bien se disuelve en la teoría luhmanniana, a partir de la forma en que se acoplan los sistemas síquicos y la sociedad. En efecto, en la concepción de Luhmann del acoplamiento individuo-sociedad, dicho vínculo supera la necesaria subordinación del individuo con respecto de la sociedad porque no son los valores ni las normas imponiéndose desde la sociedad sobre los individuos los que explican la coordinación social, sino que son las expectativas y los medios simbólicos que operan como un mecanismo abstracto que facilita y probabiliza el acoplamiento entre lo idiosincrático de los sistemas psíquicos y lo generalizado de los sociales. Dicho mecanismo permite acoplar en forma equilibrada -sin determinaciones unilaterales- las motivaciones de los individuos con la selección social, ventaja teórica ante el problemático conflacionismo descendente que afecta a la teorización parsoniana. 
En la reconceptualización de la doble contingencia es posible encontrar también una ventaja teórica por parte de la teoría luhmanniana. El concepto de doble contingencia también lo adopta Luhmann de la obra de Parsons, pero le resta las referencias a la acción en términos de fines y medios, y a la interacción como un ajuste entre éstos, cuya posibilidad era sostenida en el hecho que ambos, alter y ego, compartían un mismo sistema cultural y normativo. Parsons, cuya teoría de la sociedad parte del problema diferenciación/integración, aseguraba la complementariedad recíproca de las expectativas de alter y ego en forma de una generalización simbólica de valores. Ello, según Luhmann, no lograría dar cuenta de cómo se reproduce y amplía la contingencia de la acción individual dentro de la estructura de los sistemas sociales.

Para Luhmann, la doble contingencia describe la situación ineludible en que se encuentran alter y ego (dos sistemas psíquicos en el caso de la interacción pero la doble contingencia es también el caso cuando alter y ego son sistemas sociales) y ello plantea el problema no sólo de la factibilidad de la comunicación, sino de la sintonización de los comportamientos. Sostiene: "Todos los hechos pueden considerarse contingentes: el mundo objetivo, el Yo concreto con su biografía, la vida consciente, las decisiones, las expectativas y las otras personas con sus experiencias y elecciones. La contingencia es un universal pero, sin embargo, presupone un punto de vista subjetivo" (1998a:16). Para Luhmann la doble contingencia es a la vez subjetiva y universal. El potencial subjetivo en la interacción consiste en que tanto alter como ego, desde sus respectivas clausuras operativas, pueden negar y concebir otras posibilidades. Así, la participación de los individuos no se limita a enfrentar la doble contingencia en base a valores compartidos, sino queda incorporada teóricamente su propia y particular comprensión y posibilidades de alterar el rumbo de la comunicación.

Desde la perspectiva de la relación entre individuos encontramos en la propuesta sistémica una nueva ventaja teórica ante los conceptos tradicionales, ahora para observar cómo se ponen en relación las "subjetividades" individuales. Cuando se analiza la relación individuo-sociedad, el tema de la "inter-subjetividad" resulta insoslayable. La teoría de sistemas sociales descarta la explicación sociológica que se basa en el concepto de intersubjetividad, utilizado tanto bajo el paradigma del sujeto como en los intentos por superar los aspectos trascendentales del paradigma de la conciencia, como en el caso de Habermas. Luhmann presenta argumentos contra estas opciones teóricas y opta por el abandono total de la teoría del sujeto y del concepto de intersubjetividad.

Las insuficiencias asociadas al concepto de sujeto no son un aporte de la teoría de Luhmann; han sido tratadas exhaustivamente -por lo que no se abordarán aquí-, entre otros por Habermas, pero no así las que afectan al concepto de intersubjetividad. Al respecto, Luhmann expone las contradicciones teóricas que presenta dicho concepto, mostrando que la resolución del problema de la coordinación entre los individuos a través del concepto de intersubjetividad no logra abandonar el paradigma del sujeto. En efecto, a partir de la propia crítica que desarrolla Habermas a la teoría del sujeto, Luhmann indica que la intersubjetividad no puede ser una alternativa de la subjetividad. Sostiene que la intersubjetividad no puede considerarse un concepto en sí mismo sino que permanece atada al contexto de una teoría del sujeto, por cuanto la propuesta de lo "intersubjetivo" sólo puede plantearse respecto del concepto de sujeto. De este modo, al intentar deshacerse del sujeto en cuanto concepto central del paradigma de la conciencia, la intersubjetividad termina por desahuciarse a sí misma, concluye Luhmann (1998b:32).

A este argumento el autor agrega el que la noción de intersubjetividad tiene dificultades para indicar la unidad de aquello que es aludido con el concepto, pues se trata de un concepto relacional, y la categoría de relación tiene la desventaja de tener que dar por supuesto lo que es referido para afirmar su objeto. Por último, puesto el problema en términos lógicos, según Luhmann: "el 'inter' contradice al 'sujeto'; o dicho con más exactitud: cada sujeto tiene su propia intersubjetividad" (1998b:32).

Ante los problemas asociados al concepto de intersubjetividad, Luhmann propone remplazar el principio relacional del "inter" de las subjetividades por un principio sistémico al que le reconoce posibilidades analíticas superiores. Parte de que los sistemas, psíquicos o sociales, son autorreferentes pero a la vez se constituyen en entornos recíprocos. Ello implica que cualquier observación debe indicar cuál es la referencia sistémica de la que parte, puesto que "todo aquello que sea susceptible de indicarse como 'inter' es algo que se observa a través de límites sistémicos, 
por lo que para cada sistema es un 'inter' distinto" (1998b:40). En consecuencia, la intersubjetividad se remplaza en la propuesta luhmanniana por la teoría sistémica de la doble contingencia y la teoría de la comunicación.

Ahora, la emergencia de la comunicación, esto es la sociedad, implica un proceso de comunicación homogéneo y cerrado sólo respecto de la relación entre las selecciones, pero a la vez permanece abierto a recibir la irritación de los sistemas psíquicos situados en su entorno, como instancias que participan en dicho proceso comunicativo. "La comunicación presupone sistemas en el entorno y está preparada para la irritación constante por parte de su entorno", afirma Luhmann (2007:76). Es cierto que expresiones como ésta motivan las críticas respecto de la menor importancia otorgada a los individuos frente a las estructuras sociales. Sin embargo, no se trata de una subvaloración teórica, sino que una descripción como la anterior revela la perspectiva de observación de la sociedad, lo que implica referirse técnicamente a los individuos como su entorno.

Si, por el contrario, se observa la comunicación desde la perspectiva de los individuos, es la sociedad la que aparece como el entorno de los sistemas psíquicos que se acoplan a la comunicación. De esta manera, la sociedad constituye sólo el ambiente de los sistemas psíquicos, aunque es, a la vez, condición indispensable de la construcción de su complejidad. Pero ello no significa que se transfieran estructuras sociales a los sistemas psíquicos, los que en base a su autonomía tienen la posibilidad de rechazar los patrones sociales de conducta y ensayar otros.

Por cierto, las consecuencias de esta teoría de la comunicación implican un cambio en la concepción del proceso de socialización. El problema de la motivación para aceptar reducciones selectivas que Parsons resuelve a través de los conceptos de internalización y de socialización se resuelve por otro medio en la teorización luhmanniana. Desde esta teoría el sistema psíquico ya no puede ser penetrado por elementos pertenecientes a los sistemas sociales ni viceversa. La socialización se convierte así en una auto-socialización en tanto el sistema autopoiético de la sociedad pone su complejidad a disposición de los sistemas psíquicos auto-referentes. En sentido inverso, la inclusión es el mecanismo con que un sistema psíquico autopoiético pone la complejidad que le es propia a disposición de la construcción de sistemas sociales. Sin inclusión de los sistemas psíquicos no pueden emerger sistemas sociales, pero serán estos últimos los que luego regulen la forma en que la inclusión de los sistemas psíquicos se hace posible. En la conceptualización de Luhmann, a la vez que la (auto)socialización otorga mayores grados de autonomía a los individuos ante la sociedad, evita conferirles una natural tendencia hacia la integración social o a la conformidad con las normas sociales.

Se han destacado hasta aquí algunas "ventajas teóricas" por parte de la teoría de sistemas sociales en cuanto a ofrecer soluciones teóricas a problemas aún pendientes en la sociología. La creciente toma de conciencia por parte de las ciencias sociales de la complejidad que ha alcanzado la sociedad y el desafío de dar cuenta de ésta y su relación con el individuo, ha ido exigiendo conceptualizaciones teóricas capaces de ofrecer explicaciones acordes a dichos niveles de complejidad. La relación entre individuo y sociedad resulta un terreno apropiado para observar el nivel de cumplimento de estas exigencias por parte de las diferentes aproximaciones teóricas.

\section{A modo de síntesis y conclusión}

Se ha señalado cómo la teoría de sistemas sociales en base al concepto de emergencia, logra sortear con éxito los problemas de las distintas formas de reduccionismos, ya sea como conflacionismo ascendente, descendente o elisionismo, en que el individuo o la sociedad pierden sus propiedades, o se diluyen unas en otras. En ello se ha observado una ventaja teórica, señalándose como otra ventaja teórica la capacidad de la teoría de sistemas sociales para dar cuenta de los procesos y mecanismos involucrados en el vínculo entre el individuo y la sociedad.

Se ha argumentado que la teoría de la comunicación propuesta por Luhmann, junto a su reformulación de las teorías parsonianas de la doble contingencia y la teoría de los medios de comunicación simbólicamente generalizados, resultan ventajas teóricas ante conceptos tradicionales como cultura, socialización e internalización. Se han expuesto, asimismo, las ventajas teóricas que implica la elección de conceptos básicos como el de sistemas observadores que operan autopoiéticamente y su dinámica de acoplamientos, descartando los conceptos alternativos de sujeto e intersubjetividad. Al respecto se ha indicado que la alternativa de concebir teóricamente a 
los seres humanos como sistemas psíquicos autopoiéticos, que en sus relaciones con otros en la sociedad se atribuyen ya sea vivencias o acciones y que comprenden en base a sus propias idiosincrasias, permite describir la complejidad de los seres humanos evitando caer tanto en reduccionismos como en idealizaciones.

Se ha querido destacar en particular el que los individuos situados en el entorno de la sociedad, concebidos como capaces de operar autónoma pero acopladamente, contrario a los juicios críticos, no terminan siendo títeres en el escenario de lo social, sino que poseen cierta fuerza causal sobre la comunicación, la sociedad. Si bien se ha aclarado que la comunicación está sujeta a la autonomía de su emergencia, ello no impide que la autonomía de los individuos tenga posibilidades concretas de influir en la orientación de la comunicación. Ello significa que los individuos, más allá de ser indispensables como condición de posibilidad de la comunicación, con su presencia dan origen a los sistemas de interacción, y a través de éstos adquieren un potencial alto para irritar al orden emergente de la comunicación organizacional, incluso funcional. De este modo, aun cuando no controlen las operaciones de la sociedad, los individuos, situados en el entorno de ésta, mantienen la posibilidad de influir en ella. Contrario entonces, a lo que parece implicar la opción teórica de describir al individuo en el entorno de la sociedad, la teoría de sistemas sociales no lo deja en una situación desmedrada frente a ésta.

Como consecuencia, y lo que resulta aún más revelador desde la perspectiva de potenciales intervenciones sociales, es que la teoría de sistemas sociales indica las condiciones y posibilidades de que disponen los individuos para enfrentar las estructuras sociales. No sólo no priva a los individuos de su capacidad de incidencia, sino que por el contrario, aclara mejor los obstáculos y desafíos que dicha incidencia debe enfrentar en la compleja sociedad contemporánea.

Pero para fundamentar que el individuo cuenta con posibilidades de influir en el rumbo de la comunicación y no termina siendo títere de la sociedad, la teoría de sistemas sociales no requiere apoyarse en un humanismo positivo ni considerar al individuo un sujeto ni un actor privilegiado. Ello le permite desprenderse de "la ilusión de que la sociedad pueda organizarse de modo humano", y, así como aparta al individuo de una posición de títere de la sociedad, tampoco lo concibe como el titiritero manejando discrecionalmente los hilos de lo social.

Es indudable que bajo la descripción sociológica sistémica el ser humano pierde el ser comprendido bajo su cualidad intrínseca y suprema de ser racional, sujeto de la historia, agente privilegiado de lo que ocurre en la sociedad. Desde la perspectiva sistémica estas descripciones de los seres humanos no son sino auto-descripciones históricas producidas en la sociedad, fundamentalmente desde el ámbito filosófico, las que no necesariamente facilitan, sino más bien entorpecen la comprensión de la compleja relación individuo-sociedad. Ello lleva a considerar que son las explicaciones en base a los conceptos de la teoría de sistemas sociales, lo que facilitará a la sociología y eventualmente a otras ciencias sociales avanzar -dentro de la perspectiva científica- en la comprensión del individuo y su relación con la sociedad.

Desde el punto de vista de la investigación basada en la teoría de sistemas sociales, es posible agregar aquí que la perspectiva sistémica de concebir al individuo y a la sociedad como sistemas observadores, se constituye también en una ventaja en el ámbito de la investigación empírica. La observación de segundo orden -observar observadores- y su validación en el ámbito investigativo científico, logra además, transparentar el que las explicaciones científicas no son nada más -pero nada menos- que operaciones dentro de una sucesión autosostenida de experiencias de observación.

Por último, junto a las ventajas teóricas que se han señalado, se observa también en la teoría de sistemas sociales lo que se ha llamado una "negligencia teórica", esto es, un tratamiento desatento en el plano de la exposición de la teoría respecto del operar autónomo de los individuos y su capacidad de incidencia en la sociedad. No se trata, como se reiteró, de un desconocimiento de la contribución de los individuos a la dinámica permanente de reproducción de lo social, sino de una exposición de la perspectiva sistémica de los individuos no aparejada a la centralidad otorgada a la perspectiva sistémica de la sociedad. Ante ello este texto ha intentado aportar una discusión tomando como referencia sistémica a los individuos, referencia a la que se le concede una capacidad no sólo de aportar a la 
comprensión de la teoría, sino que también a ser fructíferamente aplicada a la investigación empírica en las ciencias sociales.

\section{Notas}

(1) Este artículo es parte del proyecto FONDECYT Postdoctoral $\mathrm{N}^{\circ} 3140077$.

(2) Luhmann renuncia al esquema de cognición sujeto/objeto, por lo que la teoría de las auto-descripciones remplaza la teoría del conocimiento que busca certezas intersubjetivas por el lado del sujeto y presupone estabilidad de los objetos. Desde el paradigma sistémico las auto-descripciones no definen el ser de los objetos, sino que aluden a descripciones que sistemas observadores (individuos, organizaciones, sistemas funcionales) realizan al comunicarse. Bajo el paradigma sistémico, la sociedad -conformada por sistemas que procesan sentido- está condenada a operar con auto-descripciones (Luhmann 2007:688).

(3) Luhmann aborda el problema filosófico de la trascendentalidad de la conciencia en diversas partes de su obra, en particular en 1991 capítulo 7, también en 2007 capítulo 1 sección IV y capítulo 5 secciones II y XIII. El autor critica el concepto trascendental de la conciencia y su diferenciación entre lo empírico y lo trascendental. Para Luhmann, el tratar a la conciencia como un sistema autopoiético logra, precisamente, suprimir la diferencia entre empírico y trascendental (1991:269).

(4) El concepto de atribución es definido por Luhmann como un mecanismo que establece suposiciones causales a las selecciones que alter o ego observan. Alter y ego representan sistemas observadores, por lo tanto no constatan realidades (no son sujetos frente a objetos) sino que sólo pueden atribuir (imputar) acciones o vivencias tanto a sí mismo como al otro.

Cuando se observa que el sistema irrita a su entorno, se atribuye como una acción (ya sea que la atribución se haga a sí mismo o al otro) y cuando se observa (no importa por quién) una irritación por parte del entorno hacia el sistema, se atribuye como una vivencia en el sistema. En otras palabras, acción es una atribución que observa la actividad del sistema, y vivencia es una atribución que expresa la recepción de una irritación que viene del entorno (Luhmann 1991, 2007).

(5) Aparentemente el concepto de persona equivaldría al concepto sociológico de rol, pero no se corresponden. La diferencia entre el concepto de rol y el de persona es que este último individualiza, mientras los roles refieren a categorías abstractas (1998b:239). Una explicación exhaustiva se encuentra en "La forma persona" en Luhmann 1998b: 231-6.

(6) Conviene aclarar que sistémicamente, los conceptos de inclusión y exclusión pierden su dimensión normativa y expresan la sola función de acoplamiento de las personas a la sociedad. De esa manera tanto inclusión como exclusión pueden sostenerse como equivalentes a "integración social" (Lockwood) en el sentido de indicar la relación entre sistemas psíquicos y sistemas sociales (Luhmann 2007:490).

\section{Bibliografía}

Archer, M. 2009. Teoría social realista, el enfoque morfogenético. Santiago de Chile: Ediciones Universidad Alberto Hurtado.

Habermas, J. 1993. El discurso filosófico de la modernidad. Madrid: Taurus.

Habermas, J. 2002. La lógica de las ciencias sociales. Madrid: Tecnos.

Izuzquiza, I. 1990. La sociedad sin hombres. Barcelona: Anthropos.

Luhmann, N. 1985. El amor como pasión. Barcelona: Ediciones Península.

Luhmann, N. 1991. Sistemas sociales. Lineamientos para una teoría general. México: Alianza Universidad.

Luhmann, N.1995. Individuo, individualidad, individualismo. Zona Abierta 70-71: 53-157.

Luhmann, N. 1998a. Teoría de los sistemas sociales (artículos). México: Universidad Iberoamericana. 
Luhmann, N. 1998b. Complejidad y modernidad. Madrid: Trotta.

Luhmann, N. 2007. La Sociedad de la sociedad. México: Herder / Universidad Iberoamericana.

Mascareño, A. 2008. Acción, estructura y emergencia en la teoría sociológica. Revista de Sociología 22: $217-256$.

Mascareño, A. 2009. Medios simbólicamente generalizados y el problema de la emergencia. Cinta moebio 36: 174197. doi: 10.4067/S0717-554X2009000300003

Miranda, P. 2012. La preconcepción de lo humano en la sociología de Luhmann. Santiago de Chile: Ediciones Universidad Alberto Hurtado.

Recibido el 15 Jul 2013

Aceptado el 5 Ene 2014 